\title{
Keto is the Hottest Weight Loss Trend this Year!
}

\section{Bennifer Bharucha*}

Nutritional Sciences, Regulations, Innovations, Scientific Intelligence, Singapore

*Corresponding Author: Bennifer Bharucha, Nutritional Sciences, Regulations, Innovations, Scientific Intelligence, Singapore.

Received: June 17, 2019; Published: July 08, 2019

DOI: 10.31080/ASNH.2019.03.0362

"I want to lose weight really fast!", "I have become so bloated with all the emotional eating!", "My wedding is next month and I need to shed 6-7kgs asap!", "I cannot get over eating loads of cheese and butter, how will I lose my weight!".

Aren't these some of the most common complaints we all have. Isn't healthy eating boring and dreadful!

So what's up and new about going on a ketogenic diet?

Well, the quick weight loss fad diet, comes with its own do's and don'ts. Let's have a look at some of the commonly googled questions about the infamous weight loss routine:

What is ketogenic diet?

The Ketogenic diet, or Keto diet, is one of the quick weight loss methods, wherein you drastically reduce your carbohydrate intake and replace it with fat in order to get your metabolism to a state called ketosis.

In order for ketosis to happen, the body needs to burn the fat stored in your body. Thereby, instead of using glucose as a fuel source to provide energy, its using the fat stores built in your body to provide the same.

This can happen in two ways

1. By fasting: Complete abstinence from food, wherein, your body converts the fat stored in your body to glucose to produce energy, thus burning the flab/extra body weight clinging to your body and making you lose your body weight.

2. Adopting the keto diet: Here in you increase your fat intake in the food and completely eliminate carbohydrates from your diet. Thereby, the body uses the stored fat in your body to produce energy rather than the carbohydrates that would be providing you with energy other- wise. Carbohydrate rich foods such as - grains, sugar and related processed food products such as bread, chips, chocolates, beverages, high calories fruits (Mango, banana, sapota, lichi).

\section{Disclaimer!!!}

Watch out for excessive fat consumption: One can also overeat on Keto in order to GAIN weight. Don't expect to eat UNLIMITED calories of cheese, butter, avocados, prawns and bacon and lose weight.

On a ketogenic diet, you can watch out for the following

- Determine your total calorie intake goal: Calculate your "basal metabolic rate" (i.e., calories you burn per day). For instance, my weight is $65 \mathrm{kgs}$, height is $170 \mathrm{cms}$, my BMR is roughly $1500 \mathrm{kcal}$. Since my activity is sedentary, my kcal consumed in the day should not be more than $1800 \mathrm{kcal}$.

- $\quad$ Remove $5 \%$ of that number for your total amount of carbs: Divide by 4 (there are 4 calories per gram of carbohydrate). Some people choose to stick to a rule of "Less than 50 grams total" or " 20 net carbs total". I have 90 calories for carbs (by calculating 5\% carbohydrates), divided by 4 , equals 22.2 grams of carbs. In my instance, I would keep a do-able target $30 \mathrm{~g}$ of carbs to start with and gradually taper that down as well.

- Next, calculate your protein requirements: Target 0.8 $1.2 \mathrm{~g}$ of protein per pound of weight. You can multiply this by 4 to see how many calories total that would be. For myself, 65Kgs, would mean,65*1.2=78g. 78g*4Kcal=312kcal.

- Left over calories as fat: $1800 \mathrm{Kcal}-(120 \mathrm{Kcal}+312 \mathrm{Kcal})$ $=1368 \mathrm{Kcal} / 9 \mathrm{Kcal}=152 \mathrm{~g}$ FAT!

- $\quad$ Put it all together, write it down, start tracking your food: My personal body macros based on calculations, you are looking at - Carbohydrate:30g, Protein=78g, Fat $=152 \mathrm{~g}$ ! (increasing fat stores by nearly 5 times). 
These macro indicator calculations, should be a good point to start with.

Benefits of keto besides weight loss: On consuming carbohydrates, your body produces insulin to deal with the increase in sugar/glucose in your bloodstream. When you minimize carbohydrate consumption, this can in turn result in less insulin production, and your body can become more insulin sensitive, which has many health benefits.

In addition to helping with weight loss, the Keto Diet has been used to treat epilepsy, Type II diabetes, polycystic ovary syndrome, improvement in neurological diseases (Parkinson's and multiple sclerosis), cancer, reduces the risk factors in both respiratory and cardiovascular diseases.

My personal suggestion: I won't blindly opt for the keto diet. As it may lead to certain hormonal disturbances. Here's what I would suggest: Be less concerned about "staying in ketosis" and instead concern yourself with how to find a nutritional strategy that fits your daily regime. If you're keen about going Keto, try it out for a short period like a month or two to see changes in your weight. If you have health concerns, discuss this with your doctor first. Take body measurements before you go on a keto diet and after a month of keto to track inch loss changes. You will have a fair idea if the diet is working for you or not. Based on that you can adjust and course correct for the next month.

The most non effective thing would be to follow the keto diet diligently then lose weight. Once you are off the keto diet, DO NOT eat liberally and leading to weight gain.

Hence, my personal suggestion is to adopt a healthy diet which conveniently and effectively fits in your lifestyle, makes you lose weight, is healthy, natural and convenient and can be adopted as a lifestyle change.

Whatever it is, I'm glad you're reading this. Let's get the weight loss going!.

Volume 3 Issue 8 August 2019

C) All rights are reserved by Bennifer Bharucha.

Citation: Bennifer Bharucha. "Keto is the Hottest Weight Loss Trend this Year!". Acta Scientific Nutritional Health 3.8 (2019): 29-30. 